PROCEEDINGS OF THE

AMERICAN MATHEMATICAL SOCIETY

Volume 135, Number 12, December 2007, Pages 3795-3803

S 0002-9939(07)09009-0

Article electronically published on September 7, 2007

\title{
ON SUPPORT VARIETIES FOR MODULES OVER COMPLETE INTERSECTIONS
}

\author{
PETTER ANDREAS BERGH
}

(Communicated by Bernd Ulrich)

\begin{abstract}
Let $(A, \mathfrak{m}, k)$ be a complete intersection of codimension $c$, and let $\tilde{k}$ be the algebraic closure of $k$. We show that every homogeneous algebraic subset of $\tilde{k}^{c}$ is the cohomological support variety of an $A$-module, and that the projective variety of a complete indecomposable maximal Cohen-Macaulay $A$-module is connected.
\end{abstract}

\section{INTRODUCTION}

Support varieties for modules over complete intersections were defined by $\mathrm{L}$. Avramov in $\mathrm{Avr}$, and L. Avramov and R.-O. Buchweitz showed in AvB that these varieties to a large extent behave precisely like the cohomological varieties of modules over group algebras of finite groups. Further illustrating this are the two main results in this paper, the first of which says that every homogeneous variety is realized as the variety of some module. The second is a version of $\mathrm{J}$. Carlson's result $\mathrm{Car}$. Theorem $1^{\prime}$ ] on varieties for modules over group algebras of finite groups. Namely, we prove that if the variety of a module decomposes as the union of two closed subvarieties having trivial intersection, then the (completion of the) minimal maximal Cohen-Macaulay approximation of the module decomposes accordingly.

Throughout this paper we let $(A, \mathfrak{m}, k)$ be a commutative Noetherian locally complete intersection; i.e., the completion $\widehat{A}$ of $A$ with respect to the $\mathfrak{m}$-adic topology is the residue ring of a regular local ring modulo an ideal generated by a regular sequence. We denote by $c$ the codimension of $A$, that is, the integer $\mu(\mathfrak{m})-\operatorname{dim} A$, where $\mu(\mathfrak{m})$ is the minimal number of generators for $\mathfrak{m}$. All modules are assumed to be finitely generated.

We now recall the definition of support varieties for modules over complete intersections; details can be found in [Avr, Section 1] and [AvB, Section 2]. Let $\widehat{A}\left[\chi_{1}, \ldots, \chi_{c}\right]$ be the polynomial ring in the $c$ commuting Eisenbud operators of cohomological degree 2 . For every $\widehat{A}$-module $X$ there is a homomorphism $\widehat{A}\left[\chi_{1}, \ldots, \chi_{c}\right]$ $\stackrel{\phi_{X}}{\longrightarrow} \operatorname{Ext}_{\widehat{A}}^{*}(X, X)$ of graded rings under which $\operatorname{Ext}_{\widehat{A}}^{*}(X, Y)$ is a finitely generated graded $\widehat{A}\left[\chi_{1}, \ldots, \chi_{c}\right]$-module for any $\widehat{A}$-module $Y$. Using the canonical isomorphism

Received by the editors June 13, 2006 and, in revised form, September 26, 2006.

2000 Mathematics Subject Classification. Primary 13C14, 13C40, 13D07, 14M10; Secondary $20 \mathrm{~J} 06$.

Key words and phrases. Complete intersections, support varieties.

(C)2007 American Mathematical Society

Reverts to public domain 28 years from publication 
$k\left[\chi_{1}, \ldots, \chi_{c}\right] \simeq \widehat{A}\left[\chi_{1}, \ldots, \chi_{c}\right] \otimes_{\widehat{A}} k$ we obtain a homomorphism $k\left[\chi_{1}, \ldots, \chi_{c}\right]$ $\stackrel{\phi_{X} \otimes 1}{\longrightarrow} \operatorname{Ext}_{\widehat{A}}^{*}(X, X) \otimes_{\widehat{A}} k$ of graded rings under which $\operatorname{Ext}_{\widehat{A}}^{*}(X, Y) \otimes_{\widehat{A}} k$ is finitely generated over $k\left[\chi_{1}, \ldots, \chi_{c}\right]$. We denote the polynomial $\operatorname{ring} k\left[\chi_{1}, \ldots, \chi_{c}\right]$ by $H$ and the graded $H$-module $\operatorname{Ext}_{\widehat{A}}^{*}(X, Y) \otimes_{\widehat{A}} k$ by $E(X, Y)$. Furthermore, we denote the sequence $\chi_{1}, \ldots, \chi_{c}$ of Eisenbud operators by $\chi$, so that $\widehat{A}[\chi]$ and $k[\chi]$ are shorthand notations for $\widehat{A}\left[\chi_{1}, \ldots, \chi_{c}\right]$ and $k\left[\chi_{1}, \ldots, \chi_{c}\right]$, respectively.

Let $M$ be an $A$-module and $\widehat{M}=\widehat{A} \otimes_{A} M$ be its m-adic completion. The support variety $\mathrm{V}(M)$ of $M$ is the algebraic set

$$
\mathrm{V}(M)=\left\{\alpha=\left(\alpha_{1}, \ldots, \alpha_{c}\right) \in \tilde{k}^{c} \mid f(\alpha)=0 \text { for all } f \in \operatorname{Ann}_{H} E(\widehat{M}, \widehat{M})\right\},
$$

where $\tilde{k}$ is the algebraic closure of $k$. This is equal to the algebraic set defined by the annihilator in $H$ of $E(\widehat{M}, k)$.

For an ideal $\mathfrak{a}$ of $H$ we denote by $\mathrm{V}_{H}(\mathfrak{a})$ the algebraic set in $\tilde{k}^{c}$ defined by a, i.e.,

$$
\mathrm{V}_{H}(\mathfrak{a})=\left\{\alpha=\left(\alpha_{1}, \ldots, \alpha_{c}\right) \in \tilde{k}^{c} \mid f(\alpha)=0 \text { for all } f \in \mathfrak{a}\right\} .
$$

Note that the variety $\mathrm{V}(M)$ of $M$ is the set $\mathrm{V}_{H}\left(\operatorname{Ann}_{H} E(\widehat{M}, \widehat{M})\right)$, and if $f$ is an element of $H$, then $\mathrm{V}_{H}(f)$ is the set of all elements in $\tilde{k}^{c}$ on which $f$ vanishes.

\section{REALIZING SUPPORT VARIETIES}

Before proving the main results we need some notation. Let $R$ be a commutative Noetherian local ring and $X$ an $R$-module with minimal free resolution

$$
\cdots \rightarrow P_{2} \rightarrow P_{1} \rightarrow P_{0} \rightarrow X \rightarrow 0,
$$

and denote by $\Omega_{R}^{n}(X)$ the $n$th syzygy of $X$. For an $R$-module $Y$, a homogeneous element $\eta \in \operatorname{Ext}_{R}^{*}(X, Y)$ can be represented by a map $f_{\eta}: \Omega_{R}^{|\eta|}(X) \rightarrow Y$, giving the pushout diagram

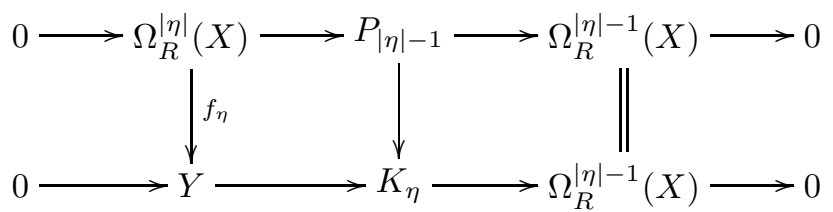

with exact rows. Note that the module $K_{\eta}$ is independent, up to isomorphism, of the map $f_{\eta}$ chosen as a representative for $\eta$. The construction of this module first appeared in the paper AGP by L. Avramov, V. Gasharov and I. Peeva, where it is used in the proof of Theorem 7.8.

If $\theta \in \operatorname{Ext}_{R}^{*}(X, X)$ is another homogeneous element, then the Yoneda product $\eta \theta \in \operatorname{Ext}_{R}^{*}(X, Y)$ is a homogeneous element of degree $|\eta|+|\theta|$. The following lemma links $K_{\eta}$ and $K_{\theta}$ to $K_{\eta \theta}$ via a short exact sequence and will be a key ingredient in the proof of the decomposition theorem in the next section.

Lemma 2.1 ([Ber, Lemma 2.3]). If $\theta \in \operatorname{Ext}_{R}^{*}(X, X)$ and $\eta \in \operatorname{Ext}_{R}^{*}(X, Y)$ are two homogeneous elements, then there exists an exact sequence

$$
0 \rightarrow \Omega_{A}^{|\eta|}\left(K_{\theta}\right) \rightarrow K_{\eta \theta} \oplus F \rightarrow K_{\eta} \rightarrow 0
$$

of $R$-modules, where $F$ is free. 
Now suppose $R$ is Gorenstein and $X$ is a maximal Cohen-Macaulay (or "MCM" from now on) module. Then there exists a complete resolution

$$
\mathbb{P}: \cdots \rightarrow P_{2} \rightarrow P_{1} \rightarrow P_{0} \stackrel{d}{\rightarrow} P_{-1} \rightarrow P_{-2} \rightarrow \cdots
$$

of $X$, i.e., a doubly infinite exact sequence of free modules in which $\operatorname{Im} d$ is isomorphic to $X$. For an integer $n \in \mathbb{Z}$ the stable cohomology module $\widehat{\operatorname{Ext}}_{R}^{n}(X, Y)$ is defined as the $n$th homology of the complex $\operatorname{Hom}_{R}(\mathbb{P}, Y)$. If $X$ and $Y$ are $\widehat{A}$ modules and $X$ is MCM, then $\widehat{\operatorname{Ext}}_{\widehat{A}}^{*}(X, Y)=\bigoplus_{i=-\infty}^{\infty} \widehat{\operatorname{Ext}}_{\widehat{A}}^{i}(X, Y)$ is a module over the ring $\widehat{A}[\chi]$ of cohomology operators, and the exact same proof as the one used to prove EHSST, Lemma 4.2] shows that for any prime ideal $\mathfrak{q} \neq(\chi)$ of $\widehat{A}[\chi]$ the $\widehat{A}[\chi]_{\mathfrak{q}}$-modules $\widehat{\operatorname{Ext}}_{\widehat{A}}^{*}(X, Y)_{\mathfrak{q}}$ and $\operatorname{Ext}_{\widehat{A}}^{*}(X, Y)_{\mathfrak{q}}$ are isomorphic.

We are now ready to prove the first result, which shows that when "cutting down" the variety of an MCM $\widehat{A}$-module by a homogeneous element, the resulting homogeneous algebraic set is also the variety of an $\widehat{A}$-module.

Theorem 2.2. Let $\eta \in H^{+}=(\chi)$ be a homogeneous element, and let $\bar{\eta} \in \widehat{A}[\chi]$ be a homogeneous element such that $\bar{\eta} \otimes 1$ corresponds to $\eta$ under the isomorphism $H \simeq \widehat{A}[\chi] \otimes_{\widehat{A}} k$. Furthermore, let $X$ be an $\widehat{A}$-module, and let $\theta \in \operatorname{Ext}_{\widehat{A}}^{*}(X, X)$ be any homogeneous element such that $\theta \otimes 1=\phi_{X}(\bar{\eta}) \otimes 1$ in $E(X, X)=\operatorname{Ext}_{\widehat{A}}^{*}(X, X) \otimes_{\widehat{A}} k$. Then there is an inclusion

$$
\mathrm{V}\left(K_{\theta}\right) \subseteq \mathrm{V}(X) \cap \mathrm{V}_{H}(\eta),
$$

and equality holds whenever $X$ is MCM.

Proof. Consider the exact sequence

$$
0 \rightarrow X \rightarrow K_{\theta} \rightarrow \Omega_{\widehat{A}}^{|\eta|-1}(X) \rightarrow 0
$$

representing $\theta$. Since varieties are invariant under syzygies we have $\mathrm{V}\left(K_{\theta}\right) \subseteq \mathrm{V}(X)$, and so the first half of the theorem will follow if we can establish the inclusion $\mathrm{V}\left(K_{\theta}\right) \subseteq \mathrm{V}_{H}(\eta)$.

The exact sequence induces a long exact sequence

$$
\begin{gathered}
0 \rightarrow \operatorname{Hom}_{\widehat{A}}\left(\Omega_{\widehat{A}}^{|\eta|-1}(X), k\right) \longrightarrow \operatorname{Hom}_{\widehat{A}}\left(K_{\theta}, k\right) \longrightarrow \operatorname{Hom}_{\widehat{A}}(X, k) \\
\stackrel{\circ \theta}{\longrightarrow} \operatorname{Ext}_{\widehat{A}}^{|\eta|}(M, k) \longrightarrow \operatorname{Ext}_{\widehat{A}}^{1}\left(K_{\theta}, k\right) \longrightarrow \operatorname{Ext}_{\widehat{A}}^{1}(X, k) \\
\stackrel{\circ(-\theta)}{\longrightarrow} \operatorname{Ext}_{\widehat{A}}^{|\eta|+1}(M, k) \longrightarrow \operatorname{Ext}_{\widehat{A}}^{2}\left(K_{\theta}, k\right) \longrightarrow \operatorname{Ext}_{\widehat{A}}^{2}(X, k) \\
\vdots \\
\stackrel{\circ(-1)^{i} \theta}{\longrightarrow} \operatorname{Ext}_{\widehat{A}}^{|\eta|+i}(M, k) \longrightarrow \operatorname{Ext}_{\widehat{A}}^{i+1}\left(K_{\theta}, k\right) \rightarrow \operatorname{Ext}_{\widehat{A}}^{i+1}(X, k)
\end{gathered}
$$

in cohomology, from which we obtain the short exact sequence

$$
\left.0 \rightarrow \frac{\operatorname{Ext}_{\widehat{A}}^{*+|\eta|}(X, k)}{\operatorname{Ext}_{\widehat{A}}^{*}(X, k) \circ \theta} \stackrel{f}{\rightarrow} \operatorname{Ext}_{\widehat{A}}^{*+1}\left(K_{\theta}, k\right) \stackrel{g}{\rightarrow} \operatorname{Ker}(\circ \theta)\right|_{\operatorname{Ext}_{\widehat{A}}^{*+1}(X, k)} \rightarrow 0 .
$$


Now for any $\widehat{A}$-modules $W$ and $Z$ the left and right scalar actions from $\widehat{A}[\chi]$ on $\operatorname{Ext}_{\widehat{A}}^{*}(W, Z)$, through the ring homomorphisms $\phi_{Z}$ and $\phi_{W}$, respectively, are actually equal (see $\underline{\mathrm{AvB}}, 1.1 .2])$. Consequently $\operatorname{Ext}_{\widehat{A}}^{*}(X, k) \circ \theta$ is an $\widehat{A}[\chi]$-submodule of $\operatorname{Ext}_{\widehat{A}}^{*+|\eta|}(X, k)$, and the above short exact sequence is a sequence of $\widehat{A}[\chi]$-modules and maps. Moreover, the end terms are both annihilated by the element $\bar{\eta}$. To see this, note that since $\theta \otimes 1=\phi_{X}(\bar{\eta}) \otimes 1$ in $\operatorname{Ext}_{\widehat{A}}^{*}(X, X) \otimes_{\widehat{A}} k$, the element $\phi_{X}(\bar{\eta})-\theta \in \operatorname{Ext}_{\widehat{A}}^{*}(X, X)$ can be written as a finite sum

$$
\phi_{X}(\bar{\eta})-\theta=\sum m_{i} \theta_{i}
$$

where $m_{i} \in \widehat{\mathfrak{m}}$ and $\theta_{i} \in \operatorname{Ext}_{\widehat{A}}^{*}(X, X)$. If $G^{*}=\bigoplus_{i=0}^{\infty} G^{i}$ is any graded right $\operatorname{Ext}_{\widehat{A}}^{*}(X, X)$-module annihilated by $\theta$, and with the property that each graded part $G^{i}$ is finitely generated over $\widehat{A}$, then

$$
\left[G^{i} \cdot \phi_{X}(\bar{\eta})\right] \otimes_{\widehat{A}} k=\left[G^{i} \cdot\left(\theta+\sum m_{i} \theta_{i}\right)\right] \otimes_{\widehat{A}} k=0 .
$$

This implies that $G^{i} \cdot \phi_{X}(\bar{\eta})$ vanishes itself; hence $\bar{\eta}$ annihilates $G^{*}$. In particular, the element $\bar{\eta}$ annihilates the end terms in the above short exact sequence.

Now for any $i \geq 0$, let $w$ be an element of $\operatorname{Ext}_{\widehat{A}}^{i}\left(K_{\theta}, k\right)$, and consider the element $\bar{\eta} \cdot w \in \operatorname{Ext}_{\widehat{A}}^{i+|\eta|}\left(K_{\theta}, k\right)$. Since $g(\bar{\eta} \cdot w)=\bar{\eta} \cdot g(w)=0$, there must exist an element $z \in \frac{\operatorname{Ext}_{A}^{*+|\eta|}(X, k)}{\operatorname{Ext}_{A}^{*}(X, k) \circ \theta}$ with the property that $\bar{\eta} \cdot w=f(z)$, giving $\bar{\eta}^{2} \cdot w=f(\bar{\eta} \cdot z)=0$. Therefore the element $\bar{\eta}^{2}$ annihilates $\operatorname{Ext}_{\widehat{A}}^{i}\left(K_{\theta}, k\right)$, and so the element $\eta^{2} \in H$ is contained in $\operatorname{Ann}_{H} E\left(K_{\theta}, k\right)$. This gives the inclusion $\mathrm{V}\left(K_{\theta}\right) \subseteq \mathrm{V}_{H}\left(\eta^{2}\right)=\mathrm{V}_{H}(\eta)$, thereby establishing the first half of the theorem. Next suppose that $X$ is MCM, and let $\mathfrak{p} \neq H^{+}$be a prime ideal of $H$ containing $\eta$ and $\operatorname{Ann}_{H} E(X, k)$. Choose a prime ideal $\overline{\mathfrak{p}} \neq(\chi)$ of $\widehat{A}[\chi]$ corresponding to $\mathfrak{p}$ and containing $\bar{\eta}$ and the annihilator of $\operatorname{Ext}_{\widehat{A}}^{*}(X, k)$, and suppose $\overline{\mathfrak{p}}$ does not contain the annihilator of $\operatorname{Ext}_{\widehat{A}}^{*}\left(K_{\theta}, k\right)$.

The exact sequence from the beginning of the proof induces a long exact sequence

$$
\cdots \rightarrow \widehat{\operatorname{Ext}}_{\widehat{A}}^{i}\left(K_{\theta}, k\right) \rightarrow \widehat{\operatorname{Ext}}_{\widehat{A}}^{i}(X, k) \stackrel{\circ(-1)^{i} \theta}{\longrightarrow} \widehat{\operatorname{Ext}}_{\widehat{A}}^{i+|\eta|}(X, k) \rightarrow \widehat{\operatorname{Ext}}_{\widehat{A}}^{i+1}\left(K_{\theta}, k\right) \rightarrow \cdots
$$

in stable cohomology, which in turn gives the exact sequence

$$
0 \rightarrow \frac{\widehat{\operatorname{Ext}}_{\widehat{A}}^{*+|\eta|-1}(X, k)}{\widehat{\operatorname{Ext}}_{\widehat{A}}^{*-1}(X, k) \circ \theta} \rightarrow \widehat{\operatorname{Ext}}_{\widehat{A}}^{*}\left(K_{\theta}, k\right),
$$

in which the index $*$ ranges over all the integers. Now let

$$
\cdots \rightarrow P_{2} \stackrel{d_{2}}{\longrightarrow} P_{1} \stackrel{d_{1}}{\longrightarrow} P_{0} \stackrel{d_{0}}{\longrightarrow} P_{-1} \stackrel{d_{-1}}{\longrightarrow} P_{-2} \rightarrow \cdots
$$

be a complete resolution of $X$, and consider the group $\widehat{\operatorname{Ext}}_{\widehat{A}}^{-n}(X, k)$ for any nonnegative integer $n$. Since $\widehat{\operatorname{Ext}}_{\widehat{A}}^{-n}(X, k)=\widehat{\operatorname{Ext}}_{\widehat{A}}^{1}\left(\operatorname{Ker} d_{-(n+2)}, k\right)=\operatorname{Ext}_{\widehat{A}}^{1}\left(\operatorname{Ker} d_{-(n+2)}, k\right)$, the ring $\widehat{A}[\chi]$ acts on $\widehat{\operatorname{Ext}}_{\widehat{A}}^{-n}(X, k)$ from both sides, and these actions coincide. Therefore $\widehat{\operatorname{Ext}}_{\widehat{A}}^{*}(X, k) \circ \theta$ is an $\widehat{A}[\chi]$-submodule of $\widehat{\operatorname{Ext}}_{\widehat{A}}^{*+|\eta|}(X, k)$, and the above short exact sequence is a sequence of $\widehat{A}[\chi]$-modules.

Now recall from the discussion prior to this theorem that $\widehat{\operatorname{Ext}}_{\widehat{A}}^{*}(W, Z)_{\bar{p}} \simeq$ $\operatorname{Ext}_{\widehat{A}}^{*}(W, Z)_{\overline{\mathfrak{p}}}$ for any $\widehat{A}$-modules $W$ and $Z$ with $W$ MCM. As $\overline{\mathfrak{p}}$ does not contain the annihilator of $\operatorname{Ext}_{\widehat{A}}^{*}\left(K_{\theta}, k\right)$, we see by localizing the above short exact sequence 
at $\overline{\mathfrak{p}}$ that $\widehat{\operatorname{Ext}}_{\widehat{A}}^{*}(X, k)_{\overline{\mathfrak{p}}}=\left[\widehat{\operatorname{Ext}}_{\widehat{A}}^{*}(X, k) \circ \theta\right]_{\overline{\mathfrak{p}}}$. Since $\theta=\phi_{X}(\bar{\eta})-\sum m_{i} \theta_{i}$ in the ring $\operatorname{Ext}_{\widehat{A}}^{*}(X, X)$, and the ideal $\overline{\mathfrak{p}}$ contains both $\bar{\eta}$ and the $m_{i}$ (it contains the element $\bar{\eta}$ by assumption, and contains the ideal $\widehat{\mathfrak{m}}$ because it corresponds to the ideal $\mathfrak{p} \subseteq H$ under the isomorphism $\left.\widehat{A}[\chi] \otimes_{\widehat{A}} k \simeq H\right)$, the $\widehat{A}(\chi)_{\overline{\mathfrak{p}}}$-module $\left[\widehat{\operatorname{Ext}}_{\widehat{A}}^{*}(X, k) \circ \theta\right]_{\overline{\mathfrak{p}}}$ must be contained in $\overline{\mathfrak{p}} \widehat{A}(\chi)_{\bar{p}} \cdot \widehat{\operatorname{Ext}}_{\widehat{A}}^{*}(X, k)_{\bar{p}}$. Consequently the inclusions

$$
\widehat{\operatorname{Ext}}_{\widehat{A}}^{*}(X, k)_{\bar{p}} \subseteq \overline{\mathfrak{p}} \widehat{A}(\chi)_{\overline{\mathfrak{p}}} \cdot \widehat{\operatorname{Ext}}_{\widehat{A}}^{*}(X, k)_{\overline{\mathfrak{p}}} \subseteq \widehat{\operatorname{Ext}}_{\widehat{A}}^{*}(X, k)_{\overline{\mathfrak{p}}}
$$

hold, and so $\widehat{\operatorname{Ext}}_{\widehat{A}}^{*}(X, k)_{\overline{\mathfrak{p}}}=\overline{\mathfrak{p}} \widehat{A}(\chi)_{\overline{\mathfrak{p}}} \cdot \widehat{\operatorname{Ext}}_{\widehat{A}}^{*}(X, k)_{\overline{\mathfrak{p}}}$. But $\widehat{\operatorname{Ext}}_{\widehat{A}}^{*}(X, k)_{\overline{\mathfrak{p}}}$, being isomorphic to $\operatorname{Ext}_{\widehat{A}}^{*}(X, k)_{\bar{p}}$, is finitely generated over $\widehat{A}[\chi]_{\bar{p}}$; hence Nakayama's Lemma implies $\operatorname{Ext}_{\widehat{A}}^{*}(X, k)_{\overline{\mathfrak{p}}}=0$. This contradicts the assumption that $\overline{\mathfrak{p}}$ contains the annihilator of $\operatorname{Ext}_{\widehat{A}}^{*}(X, k)$, and therefore $\overline{\mathfrak{p}}$ must contain the annihilator of $\operatorname{Ext}_{\widehat{A}}^{*}\left(K_{\theta}, k\right)$. But then $\operatorname{Ann}_{H} E\left(K_{\theta}, k\right) \subseteq \mathfrak{p}$, giving the inclusion

$$
\sqrt{\mathrm{Ann}_{H} E\left(K_{\theta}, k\right)} \subseteq \sqrt{\left(\eta, \operatorname{Ann}_{H} E(X, k)\right)}
$$

of ideals in $H$, and consequently we get $\mathrm{V}(X) \cap \mathrm{V}_{H}(\eta) \subseteq \mathrm{V}\left(K_{\theta}\right)$.

Suppose now that we start with an $A$-module $M$, and consider its completion $\widehat{M}$. Let $\eta \in H^{+}$be a homogeneous element, let $\bar{\eta} \in \widehat{A}[\chi]$ be a corresponding element,

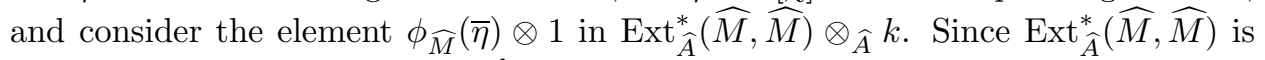
isomorphic to $\operatorname{Ext}_{A}^{*}(M, M) \otimes_{A} \widehat{A}$, there is an isomorphism

$$
\operatorname{Ext}_{A}^{*}(M, M) \otimes_{A} k \stackrel{\sim}{\longrightarrow} \operatorname{Ext}_{\widehat{A}}^{*}(\widehat{M}, \widehat{M}) \otimes_{\widehat{A}} k
$$

under which the image of an element $\theta \otimes 1 \in \operatorname{Ext}_{A}^{*}(M, M) \otimes_{A} k$ is $\widehat{\theta} \otimes 1$. Hence there exists a homogeneous element $\theta^{M} \in \operatorname{Ext}_{A}^{|\eta|}(M, M)$ such that $\widehat{\theta^{M}} \otimes 1$ equals the element $\phi_{\widehat{M}}(\bar{\eta}) \otimes 1$ in $\operatorname{Ext}_{\widehat{A}}^{*}(\widehat{M}, \widehat{M}) \otimes_{\widehat{A}} k$. Now if $\theta^{M}$ is represented by the exact sequence

$$
0 \rightarrow M \rightarrow K_{\theta^{M}} \rightarrow \Omega_{A}^{|\eta|-1}(M) \rightarrow 0,
$$

then its completion $\widehat{\theta^{M}}$ is represented by the sequence

$$
0 \rightarrow \widehat{M} \rightarrow \widehat{K_{\theta^{M}}} \rightarrow \Omega_{\widehat{A}}^{|\eta|-1}(\widehat{M}) \rightarrow 0,
$$

whose middle term is the completion of an A-module. By Theorem 2.2 the inclusion

$$
\mathrm{V}\left(K_{\theta^{M}}\right) \subseteq \mathrm{V}(M) \cap \mathrm{V}_{H}(\eta)
$$

holds, with equality holding whenever $M$ is MCM, and consequently we obtain the following corollary, showing that every homogeneous algebraic set in $\tilde{k}^{c}$ is the variety of an MCM $A$-module.

Corollary 2.3. Every closed homogeneous variety in $\tilde{k}^{c}$ is the variety of some MCM A-module.

Proof. Let $\eta_{1}, \ldots, \eta_{t}$ be homogeneous elements in $H^{+}$, and denote by $M$ the MCM module $\Omega_{A}^{\operatorname{dim} A}(k)$. Then $\mathrm{V}(M)=\mathrm{V}(k)=\tilde{k}^{c}$, and from the theorem and the above discussion we see that there exists a homogeneous element $\theta_{1} \in \operatorname{Ext}_{A}^{\left|\eta_{1}\right|}(M, M)$ with the property that $\mathrm{V}\left(K_{\theta_{1}}\right)=\mathrm{V}(M) \cap \mathrm{V}_{H}\left(\eta_{1}\right)=\mathrm{V}_{H}\left(\eta_{1}\right)$. Repeating the process with $\eta_{2}, \ldots, \eta_{t}$ we end up with an MCM $A$-module $K$ such that

$$
\mathrm{V}(K)=\mathrm{V}_{H}\left(\eta_{1}\right) \cap \cdots \cap \mathrm{V}_{H}\left(\eta_{t}\right)=\mathrm{V}_{H}\left(\eta_{1}, \ldots, \eta_{t}\right) .
$$


Remarks. (i) In an unpublished preprint (as of December 2006), L. Avramov and D. Jorgensen obtain a different proof of Corollary 2.3, based on a result concerning the realization of certain graded modules as cohomology modules. L. Avramov reported it at a meeting at MSRI, Berkeley, in December 2002.

(ii) In [EHSST] a realization theorem is proved for finite-dimensional algebras, and this result applies to complete intersections containing a field (see also $\mathrm{SnS}$, Section 7]).

\section{Decomposition}

Before proving the next result, recall that an $M C M$-approximation of an $A$ module $X$ is an exact sequence

$$
0 \rightarrow Y_{X} \rightarrow C_{X} \stackrel{f}{\rightarrow} X \rightarrow 0,
$$

where $C_{X}$ is MCM and $Y_{X}$ has finite injective dimension. The approximation is minimal if the map $f$ is right minimal, that is, if every map $C_{X} \stackrel{g}{\rightarrow} C_{X}$ satisfying $f=f g$ is an isomorphism. This notion was introduced in $\mathrm{AuB}$, where it was shown that every finitely generated module over a commutative Noetherian ring admitting a dualizing module has an MCM-approximation. Moreover, it follows from the remark following [Mar, Theorem 18] that every finitely generated module over a commutative local Gorenstein ring has a minimal MCM-approximation, which is unique up to isomorphism. In particular this applies to our setting, where $A$ is a local complete intersection. Furthermore, since $A \rightarrow \widehat{A}$ is a faithfully flat local homomorphism, an $A$-module $Z$ has finite projective dimension if and only if the $\widehat{A}$-module $\widehat{Z}$ has finite projective dimension, and it follows from Mat, Theorem 23.3 ] that $Z$ is MCM if and only if $\widehat{Z}$ is MCM. Therefore, by Mar, Proposition 19] and the fact that over a Gorenstein ring the modules having finite injective dimension are precisely those having finite projective dimension, we see that

$$
0 \rightarrow Y_{X} \rightarrow C_{X} \stackrel{f}{\rightarrow} X \rightarrow 0
$$

is a minimal MCM-approximation if and only if

$$
0 \rightarrow \widehat{Y}_{X} \rightarrow \widehat{C}_{X} \stackrel{\widehat{f}}{\rightarrow} \widehat{X} \rightarrow 0
$$

is a minimal MCM-approximation.

We are now ready to prove the second main result. It is the commutative complete intersection version of J. Carlson's famous theorem (see [Car]) from modular representation theory; if the variety $V$ of a $k G$-module $L$ (where $k$ is an algebraically closed field and $G$ is a finite group) decomposes as $V=V_{1} \cup V_{2}$, where $V_{1}$ and $V_{2}$ are closed varieties having trivial intersection, then $L$ decomposes as $L=L_{1} \oplus L_{2}$, where the variety of $L_{i}$ is $V_{i}$. Our proof follows closely that of J. Carlson, but with some adjustments.

Theorem 3.1. If for an $A$-module $M$ we have $\mathrm{V}(M)=V_{1} \cup V_{2}$, where $V_{1}$ and $V_{2}$ are closed homogeneous varieties having trivial intersection, then the completion $\widehat{C}_{M}$ of the minimal MCM-approximation of $M$ decomposes as $\widehat{C}_{M}=C_{1} \oplus C_{2}$ with $\mathrm{V}\left(C_{i}\right)=V_{i}$.

Proof. Let

$$
0 \rightarrow Y \rightarrow C \rightarrow M \rightarrow 0
$$


be the minimal MCM-approximation of $M$. Since $Y$ has finite injective dimension (or equivalently, finite projective dimension), it follows from AvB, Theorem 5.6] that $\mathrm{V}(Y)$ is trivial and that we therefore have $\mathrm{V}(M)=\mathrm{V}(C)$. Moreover, by definition the equality $\mathrm{V}(X)=\mathrm{V}(\widehat{X})$ holds for every $A$-module $X$, and therefore we may suppose that $A$ is complete.

We argue by induction on the integer $\operatorname{dim} V_{1}+\operatorname{dim} V_{2}$. If one of $V_{1}$ and $V_{2}$, say $V_{2}$, is zero dimensional, then $V_{2}$ is trivial, and the decomposition $C=C^{\prime} \oplus P$, with $P$ being the maximal projective summand of $C$, satisfies the conclusion of the theorem. Suppose therefore that $\operatorname{dim} V_{i}$ is nonzero for $i=1,2$.

Let $\mathfrak{a}_{1}$ and $\mathfrak{a}_{2}$ be homogeneous ideals of $H=k[\chi]$ defining the varieties $V_{1}$ and $V_{2}$, i.e., $V_{i}$ is the algebraic set $\mathrm{V}_{H}\left(\mathfrak{a}_{i}\right)$ in $\tilde{k}^{c}$ defined by $\mathfrak{a}_{i}$ for $i=1,2$. We then have the equalities

$$
\{0\}=V_{1} \cap V_{2}=\mathrm{V}_{H}\left(\mathfrak{a}_{1}\right) \cap \mathrm{V}_{H}\left(\mathfrak{a}_{2}\right)=\mathrm{V}_{H}\left(\mathfrak{a}_{1}+\mathfrak{a}_{2}\right),
$$

and so it follows from Hilbert's Nullstellensatz that for each $1 \leq i \leq c$ we have $\chi_{i} \in \sqrt{\mathfrak{a}_{1}+\mathfrak{a}_{2}}$. Therefore $\sqrt{\mathfrak{a}_{1}+\mathfrak{a}_{2}}$ is the graded maximal ideal $H^{+}$of $H$, i.e., $\sqrt{\mathfrak{a}_{1}+\mathfrak{a}_{2}}=(\chi)$.

Pick a homogeneous element $\theta \in H^{+}$with the property that $\operatorname{dim} H /\left(\mathfrak{a}_{2}, \theta\right)<$ $\operatorname{dim} H / \mathfrak{a}_{2}$ (this is possible since $\operatorname{dim} H / \mathfrak{a}_{2}=\operatorname{dim} V_{2}>0$ ). By the above there is an integer $n \geq 1$ such that $\theta^{n}$ belongs to $\mathfrak{a}_{1}+\mathfrak{a}_{2}$, i.e., $\theta^{n}=\theta_{1}+\eta$, where $\theta_{1} \in \mathfrak{a}_{1}$ and $\eta \in \mathfrak{a}_{2}$. Then $\operatorname{dim} H /\left(\mathfrak{a}_{2}, \theta_{1}\right)<\operatorname{dim} H / \mathfrak{a}_{2}$, which translates to the language of varieties as $\operatorname{dim}\left(\mathrm{V}_{H}\left(\mathfrak{a}_{2}\right) \cap \mathrm{V}_{H}\left(\theta_{1}\right)\right)=\operatorname{dim} \mathrm{V}_{H}\left(\mathfrak{a}_{2}+\left(\theta_{1}\right)\right)<\operatorname{dim} \mathrm{V}_{H}\left(\mathfrak{a}_{2}\right)$. Similarly we can find an element $\theta_{2} \in \mathfrak{a}_{2}$ having the property that it "cuts down" the variety defined by $\mathfrak{a}_{1}$. Hence the two homogeneous elements $\theta_{1}$ and $\theta_{2}$ satisfy

$$
\begin{aligned}
& \theta_{1} \in \mathfrak{a}_{1}, \quad \operatorname{dim}\left(V_{2} \cap \mathrm{V}_{H}\left(\theta_{1}\right)\right)<\operatorname{dim} V_{2}, \\
& \theta_{2} \in \mathfrak{a}_{2}, \quad \operatorname{dim}\left(V_{1} \cap \mathrm{V}_{H}\left(\theta_{2}\right)\right)<\operatorname{dim} V_{1} .
\end{aligned}
$$

Now since $\mathrm{V}_{H}\left(\theta_{1} \theta_{2}\right)=\mathrm{V}_{H}\left(\theta_{1}\right) \cup \mathrm{V}_{H}\left(\theta_{2}\right) \supseteq V_{1} \cup V_{2}=\mathrm{V}(C)$, it follows once more from Hilbert's Nullstellensatz that $\theta_{1} \theta_{2} \in \sqrt{\operatorname{Ann}_{H} E(C, C)}$, where $E(C, C)=$ $\operatorname{Ext}_{A}^{*}(C, C) \otimes_{A} k$. Replacing $\theta_{1}$ and $\theta_{2}$ by suitable powers, we may assume that $\theta_{1} \theta_{2} \in \operatorname{Ann}_{H} E(C, C)$. Viewed as elements in $A[\chi] \otimes_{A} k$ we have $\theta_{i}=\bar{\theta}_{i} \otimes$ 1 , where $\bar{\theta}_{1}$ and $\bar{\theta}_{2}$ are homogeneous elements of positive degrees in $A[\chi]$ with the property that $\bar{\theta}_{1} \bar{\theta}_{2} \in \operatorname{Ann}_{A[\chi]} \operatorname{Ext}_{A}^{*}(C, C)$. To see the latter, note that $0=$ $\theta_{1} \theta_{2}\left(\operatorname{Ext}_{A}^{i}(C, C) \otimes_{A} k\right)=\bar{\theta}_{1} \bar{\theta}_{2} \operatorname{Ext}_{A}^{i}(C, C) \otimes_{A} k$ for every $i \geq 0$, and since $\bar{\theta}_{1} \bar{\theta}_{2} \operatorname{Ext}_{A}^{i}(C, C)$ is a finitely generated $A$-module $\left(\bar{\theta}_{1} \bar{\theta}_{2}\right.$ commutes with elements in $A$ ), the claim follows.

Now consider the images $\theta_{1}^{C}$ and $\theta_{2}^{C}$ of $\bar{\theta}_{1}$ and $\bar{\theta}_{2}$ in $\operatorname{Ext}_{A}^{*}(C, C)$. Since $\theta_{1}^{C} \theta_{2}^{C}=0$, the bottom exact sequence in the exact commutative diagram

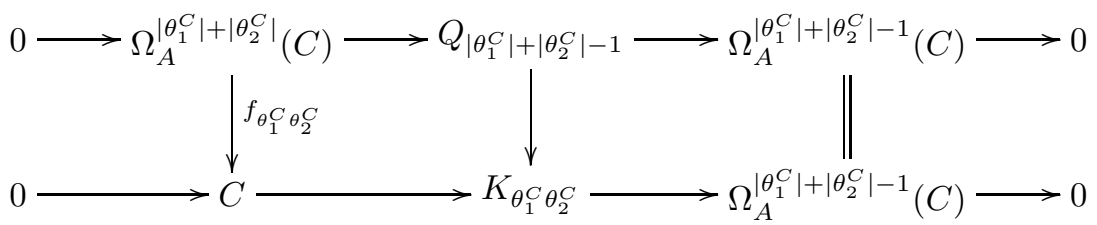

splits, where $Q_{n}$ denotes the $n$th module in the minimal free resolution of $C$. Therefore $K_{\theta_{1}^{C} \theta_{2}^{C}}$ is isomorphic to $C \oplus \Omega_{A}^{\left|\theta_{1}^{C}\right|+\left|\theta_{2}^{C}\right|-1}(C)$, and from Lemma 2.1 we see that 
there exists an exact sequence

$$
0 \rightarrow \Omega_{A}^{\left|\theta_{1}^{C}\right|}\left(K_{\theta_{2}^{C}}\right) \rightarrow C \oplus \Omega_{A}^{\left|\theta_{1}^{C}\right|+\left|\theta_{2}^{C}\right|-1}(C) \oplus F \rightarrow K_{\theta_{1}^{C}} \rightarrow 0
$$

for some free module $F$. From Theorem 2.2 we have $\mathrm{V}\left(K_{\theta_{i}^{C}}\right)=\mathrm{V}(C) \cap \mathrm{V}_{H}\left(\theta_{i}\right)$; hence the equality $\mathrm{V}(C)=V_{1} \cup V_{2}$ and the inclusion $V_{i} \subseteq \mathrm{V}_{H}\left(\theta_{i}\right)$ give the equalities

$$
\begin{aligned}
& \mathrm{V}\left(K_{\theta_{1}^{C}}\right)=V_{1} \cup\left(V_{2} \cap \mathrm{V}_{H}\left(\theta_{1}\right)\right), \\
& \mathrm{V}\left(K_{\theta_{2}^{C}}\right)=V_{2} \cup\left(V_{1} \cap \mathrm{V}_{H}\left(\theta_{2}\right)\right) .
\end{aligned}
$$

By induction there exist $A$-modules $X_{1}, X_{2}, Y_{1}$ and $Y_{2}$ such that $K_{\theta_{1}^{C}}=X_{1} \oplus X_{2}$ and $\Omega_{A}^{\left|\theta_{1}^{C}\right|}\left(K_{\theta_{2}^{C}}\right)=Y_{1} \oplus Y_{2}$, and such that

$$
\begin{aligned}
\mathrm{V}\left(X_{1}\right) & =V_{1}, \\
\mathrm{~V}\left(X_{2}\right) & =V_{2} \cap \mathrm{V}_{H}\left(\theta_{1}\right), \\
\mathrm{V}\left(Y_{1}\right) & =V_{1} \cap \mathrm{V}_{H}\left(\theta_{2}\right), \\
\mathrm{V}\left(Y_{2}\right) & =V_{2} .
\end{aligned}
$$

Now since $\mathrm{V}\left(X_{1}\right) \cap \mathrm{V}\left(Y_{2}\right)$ and $\mathrm{V}\left(X_{2}\right) \cap \mathrm{V}\left(Y_{1}\right)$ are contained in $V_{1} \cap V_{2}$, which is trivial, we see from $\mathrm{AvB}$, Theorem 5.6] that $\operatorname{Ext}_{A}^{i}\left(X_{1}, Y_{2}\right)$ and $\operatorname{Ext}_{A}^{i}\left(X_{2}, Y_{1}\right)$ vanish for $i \gg 0$. But $K_{\theta_{1}^{C}}$ is MCM, implying that $X_{1}$ and $X_{2}$ are both MCM, and so it follows from [ArY, Theorem 4.2] that $\operatorname{Ext}_{A}^{i}\left(X_{1}, Y_{2}\right)$ and $\operatorname{Ext}_{A}^{i}\left(X_{2}, Y_{1}\right)$ vanish for $i \geq 1$. Therefore

$$
\operatorname{Ext}_{A}^{1}\left(K_{\theta_{1}^{C}}, \Omega_{A}^{\left|\theta_{1}^{C}\right|}\left(K_{\theta_{2}^{C}}\right)\right)=\operatorname{Ext}_{A}^{1}\left(X_{1}, Y_{1}\right) \oplus \operatorname{Ext}_{A}^{1}\left(X_{2}, Y_{2}\right)
$$

and this implies that the exact sequence $(\mathbb{T})$ is equivalent to the direct sum of two sequences of the form

$$
0 \rightarrow Y_{i} \rightarrow Z_{i} \rightarrow X_{i} \rightarrow 0
$$

for $i=1,2$, where $Z_{i}$ is an $A$-module. Then $C \oplus \Omega_{A}^{\left|\theta_{1}^{C}\right|+\left|\theta_{2}^{C}\right|-1}(C) \oplus F$ must be isomorphic to $Z_{1} \oplus Z_{2}$, and since $\mathrm{V}\left(Z_{i}\right) \subseteq \mathrm{V}\left(X_{i}\right) \cup \mathrm{V}\left(Y_{i}\right) \subseteq V_{i}$ and the KrullSchmidt property holds for the category of (finitely generated) modules over a complete local ring, there must exist $A$-modules $C_{1}$ and $C_{2}$ such that $C=C_{1} \oplus C_{2}$ and $\mathrm{V}\left(C_{i}\right)=\mathrm{V}\left(Z_{i}\right)$. Since

$$
V=\mathrm{V}\left(C_{1}\right) \cup \mathrm{V}\left(C_{2}\right) \subseteq V_{1} \cup V_{2}=V,
$$

we must have $\mathrm{V}\left(C_{i}\right)=V_{i}$, and the proof is complete.

Corollary 3.2. The projective variety of a complete indecomposable MCM Amodule is connected.

\section{ACKNOWLEDGMENTS}

I would like to express my gratitude to Lucho Avramov for numerous comments and improvements. Also, I would like to thank Dave Jorgensen and my supervisor Øyvind Solberg for valuable suggestions and comments on this paper. 


\section{REFERENCES}

[AuB] M. Auslander, R.-O. Buchweitz, The homological theory of maximal Cohen-Macaulay approximations, Mém. Soc. Math. France 38 (1989), 5-37. MR1044344 (91h:13010)

[Avr] L. Avramov, Modules of finite virtual projective dimension, Invent. Math. 96 (1989), 71-101. MR981738 (90g:13027)

[AvB] L. Avramov, R.-O. Buchweitz, Support varieties and cohomology over complete intersections, Invent. Math. 142 (2000), 285-318. MR1794064(2001j:13017)

[AGP] L. Avramov, V. Gasharov, I. Peeva, Complete intersection dimension, Publ. Math. I.H.E.S. 86 (1997), 67-114. MR.1608565 (99c:13033)

[ArY] T. Araya, Y. Yoshino, Remarks on a depth formula, a grade inequality and a conjecture of Auslander, Comm. Algebra 26 (1998), 3793-3806. MR1647079 (99h:13010)

[Ber] P.A. Bergh, Modules with reducible complexity, J. Algebra 310 (2007), no. 1, 132-147.

[Car] J. Carlson, The variety of an indecomposable module is connected, Invent. Math. 77 (1984), 291-299. MR752822 (86b:20009)

[EHSST] K. Erdmann, M. Holloway, N. Snashall, Ø. Solberg, R. Taillefer, Support varieties for selfinjective algebras, $K$-theory 33 (2004), 67-87. MR.2199789

[Mar] A. Martsinkovsky, Cohen-Macaulay modules and approximations, in Trends in Mathematics: Infinite Length Modules, H. Krause and C. Ringel (eds.), Birkhäuser Verlag (2000), 167-192. MR1789215 (2001i:13011)

[Mat] H. Matsumura, Commutative ring theory, Cambridge University Press, 2000. MR879273 (88h:13001)

[SnS] N. Snashall, Ø. Solberg, Support varieties and Hochschild cohomology rings, Proc. London Math. Soc. 88 (2004), 705-732. MR2044054 (2005a:16014)

Institutt for matematiske fag, NTNU, N-7491 Trondheim, Norway

E-mail address: bergh@math.ntnu.no 